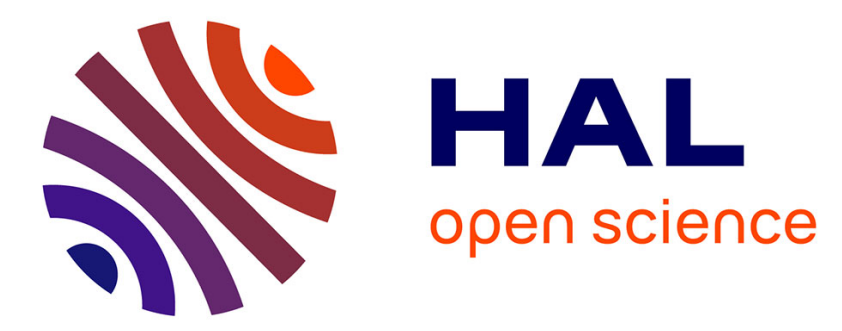

\title{
Propagation of nondiffracting pulses carrying orbital angular momentum at microwave frequencies
}

D. Comite, W. Fuscaldo, S.C. Pavone, G. Valerio, M. Ettorre, M. Albani, A. Galli

\section{- To cite this version:}

D. Comite, W. Fuscaldo, S.C. Pavone, G. Valerio, M. Ettorre, et al.. Propagation of nondiffracting pulses carrying orbital angular momentum at microwave frequencies. Applied Physics Letters, 2017, 110 (11), pp.114102. 10.1063/1.4978601 . hal-01508043

HAL Id: hal-01508043

https://hal-univ-rennes1.archives-ouvertes.fr/hal-01508043

Submitted on 13 Mar 2020

HAL is a multi-disciplinary open access archive for the deposit and dissemination of scientific research documents, whether they are published or not. The documents may come from teaching and research institutions in France or abroad, or from public or private research centers.
L'archive ouverte pluridisciplinaire HAL, est destinée au dépôt et à la diffusion de documents scientifiques de niveau recherche, publiés ou non, émanant des établissements d'enseignement et de recherche français ou étrangers, des laboratoires publics ou privés. 


\title{
Propagation of Nondiffracting Pulses Carrying Orbital Angular Momentum at Microwave Frequencies
}

\author{
D. Comite, ${ }^{1}$ W. Fuscaldo, ${ }^{1,2}$ S. C. Pavone, ${ }^{3}$ G. Valerio, ${ }^{4}$ M. Ettorre, ${ }^{2}$ M. Albani, ${ }^{3}$ and A. Galli ${ }^{1}$ \\ 1) Dipartimento di Ingegneria dell'Informazione, Elettronica e Telecomunicazioni, \\ Università degli Studi di Roma Sapienza, via Eudossiana 18, 00184, Roma, Italy. ${ }^{\text {a) }}$ \\ ${ }^{2)}$ Institut d'Électronique et de Télécommunications de Rennes (IETR), UMR CNRS 6164, Université de Rennes 1, \\ 35042 Rennes, France. \\ ${ }^{3)}$ Dipartimento di Ingegneria dell'Informazione e Scienze Matematiche, Università degli Studi di Siena, via Roma 56, \\ 53100, Siena, Italy. ${ }^{\mathrm{b})}$ \\ 4) Sorbonne Universités, UPMC Univ Paris 06, UR2, L2E, F-75005, Paris, France
}

(Dated: 30 June 2017)

We discuss the generation and propagation of nondiffracting twisted pulses at microwaves, obtained through polychromatic spectral superposition of higher-order Bessel beams. The inherent vectorial structure of Maxwell's equations has been considered to generalize the nondiffracting solution of the scalar wave equation with azimuthal phase variation. Since a wide frequency bandwidth is necessary to synthesize time-limited pulses, the non-negligible wavenumber frequency dispersion, which commonly affects propagation in the microwave range, has been taken into account. To this purpose a higher-order Bessel beam is generated by enforcing an inward cylindrical traveling-wave distribution over a finite aperture. We present and discuss the main aspects of the generation of twisted pulses in the microwave range, showing the promising possibility to carry orbital angular momentum through highly-focused X-shaped pulses up to the nondiffractive range.

PACS numbers: 63.20.Pw, 84.40.Ba, 41.20.Jb, 41.20.-q, 84.40.-x

The exponential growth of high-demanding technologies for microwave and millimeter-wave applications such as high data-rate communications, wireless power transfer, near-field probing, medical imaging, just to mention a few, has recently pushed researchers towards the recognition of novel challenging issues from the electromagnetic viewpoint: the generation of localized beams ${ }^{1,2}$ and pulses $^{3}$, as well as the generation of vortex beams ${ }^{4}$ and pulses carrying orbital angular momentum $(\mathrm{OAM})^{5}$.

As is known, localized beams are nondiffractive monochromatic solutions of the scalar wave equation, whose most-known representatives are the Bessel beams; as a consequence, they are not subject to transverse spreading, motivating the growing interest in their experimental characterization. Despite they were theoretically predicted for the first time in the $40 \mathrm{~s}^{6}$, Bessel beams have been experimentally generated in optics only at the end of the $80 \mathrm{~s}^{7}$, whereas realizations at lower frequencies appeared only in the 90 s by means of axicons ${ }^{8,9}$ and several years later by means of other techniques ${ }^{10-13}$. This temporary lack was mainly due to the fact that ideal Bessel beams are endowed by infinite energy, thus they require infinite radiating apertures ${ }^{14}$ to be generated. However, the pioneering work of Durnin ${ }^{1,7}$ revealed that such beams can still be generated by truncated apertures, although their nondiffractive behavior is limited to a certain distance, known as depth of field or nondiffractive range (NDR) (see Fig. 1).

Nondiffracting Bessel beams can profitably be used as

\footnotetext{
a) Electronic mail: \{davide.comite, walter.fuscaldo\}@uniroma1.it

b) Electronic mail: santi.pavone@unisi.it
}

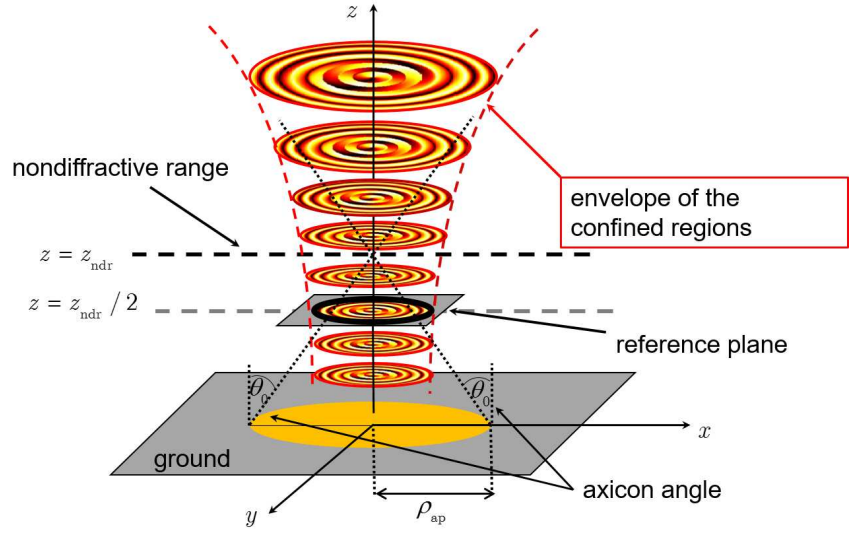

FIG. 1. Circular aperture over the $x y$ plane for launching localized waves at microwaves. Their section slowly increases beyond the nondiffractive range due to the limited spatiotemporal dispersion of the pulse. A transverse reference plane at $z_{0}=z_{\mathrm{NDR}} / 2$ is defined to observe the pulse propagation.

building blocks for the synthesis of polychromatic solutions of Helmholtz equation. Among them, localized pulses $^{2,15}$ undoubtedly gained increasing importance because of their remarkable properties of spatial and temporal confinement. It is worth noting that in the literature such solutions are also referred as focus wave modes ${ }^{16,17}$, splash pulses ${ }^{18}$, slingshot pulses ${ }^{19}$, undistorted progressive pulses ${ }^{20}$, as well as complex source wave-fields ${ }^{21,22}$, although they represent only different classes of the wider family of localized waves; a useful and clarifying comparative table is reported in Ref. 23. Such localized pulses were theoretically predicted at the beginning of 
the $80 \mathrm{~s}^{16}$, but their experimental realizations (specifically, a class of them widely known as $X$-waves) appeared only in the 90s in acoustics ${ }^{3}$ and optics ${ }^{24}$. However, there is still no experimental evidence of such $\mathrm{X}$-waves in the microwave range where the wavenumber frequency dispersion ${ }^{25}$ severely affects their propagation. This is especially true when a considerable frequency range is used to synthesize a highly-focused pulse (i.e., a pulse whose main spot is strongly confined along both the longitudinal and the transverse direction with respect to the axis of propagation). Interestingly, a recent analysis ${ }^{25}$ has shown that a class of wideband antennas, namely the radial line slot arrays (RLSA) (see Refs. 26 and 27 for a description of the structure), are able to generate highlyfocused localized pulses, provided that certain conditions regarding the wideband capability, the size of the aperture, and the wavenumber dispersion are fulfilled.

However, most of the current literature considers the generation of $\mathrm{X}$-waves as superpositions of zeroth-order Bessel beams, thus neglecting the possibility to generate polychromatic higher-order beams (also known as higherorder $\mathrm{X}$-waves ${ }^{2}$ ) that intrinsically carry orbital angular momentum (OAM). Such a feature is of particular interest in different areas of applied physics ${ }^{28}$, especially in the context of optical trapping and micro-manipulation of multiple particles ${ }^{29-32}$.

The main results regarding the generation of twisted localized pulses (i.e., higher-order localized pulses) have hitherto been achieved at optical frequencies ${ }^{5,14}$, whereas realizations and even theoretical discussions at microwaves and millimeter waves are still lacking. In this Letter, following the recent investigations about the combination of OAM and $\mathrm{X}$-waves in optics ${ }^{5}$, we investigate the possibility to generate higher-order nondiffracting pulses at microwaves. In particular, we discuss the possibility to produce limited-diffraction twisted $\mathrm{X}$-waves able to carry OAM (here synthetically referred as XOAMs) at microwaves through the aperture field potentially supported by planar antennas (e.g., RLSA antennas). Numerical results corroborate the proposed analysis.

A vectorial formulation for higher-order Bessel beams is adopted in the following. A time dependence of the kind $e^{j \omega t}$ is assumed and suppressed. Without loss of generality, a transverse magnetic field with respect to the longitudinal direction $\left(\mathrm{TM}^{z}\right)$ is considered (see Fig. 1), so that in a cylindrical reference frame $(\rho, \phi, z)$, the electric field components can be expressed as

$$
\begin{aligned}
& E_{z}(\rho, \phi, z)=E_{0} J_{n}\left(k_{\rho} \rho\right) e^{-j n \phi} e^{-j k_{z} z}, \\
& E_{\rho}(\rho, \phi, z)=-j \frac{k_{z}}{k_{\rho}} E_{0} J_{n}^{\prime}\left(k_{\rho} \rho\right) e^{-j n \phi} e^{-j k_{z} z}, \\
& E_{\phi}(\rho, \phi, z)=-\frac{n k_{z}}{k_{\rho}^{2}} E_{0} \frac{J_{n}\left(k_{\rho} \rho\right)}{\rho} e^{-j n \phi} e^{-j k_{z} z},
\end{aligned}
$$

where $J_{n}(\cdot)$ is the $n$-th order Bessel function of first kind and $J_{n}^{\prime}(\cdot)$ the first-order derivative; $k_{\rho}$ and $k_{z}=\sqrt{k_{0}^{2}-k_{\rho}^{2}}$ are the radial and longitudinal wavenumbers, respectively. The evaluation of (1)-(3) at $z=0$

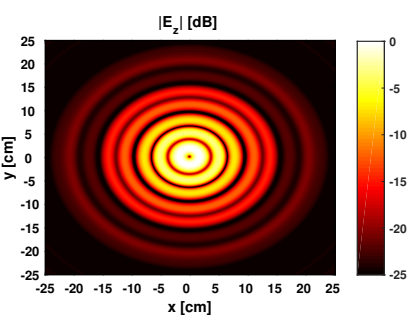

(a)

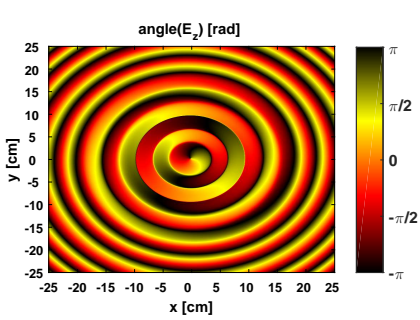

(b)
FIG. 2. XOAM wave: $E_{z}$ radiated over a transverse plane $\left(z_{0}=30 \mathrm{~cm}\right)$ at the carrier frequency $f=12.5 \mathrm{GHz}$. (a) normalized amplitude and (b) phase distribution.

(i.e., on the antenna-aperture plane) gives the equivalent tangential electric field distribution to be synthesized. Such a standing-wave distribution is inherently narrow-band ${ }^{10}$, whilst the generation of efficiently confined localized pulses requires wideband capabilities, as discussed in detail in Ref. 25. However, it has recently been demonstrated ${ }^{27}$ that an inward traveling-wave aperture distribution can generate a Bessel beam in a biconical region close to the axis of symmetry of the aperture. Hence, in order to design a wideband launcher, we synthesize an inward cylindrical traveling-wave aperture distribution for the tangential electric field by replacing the $J_{n}^{\prime}, J_{n}$ functions in Eqs. (2)-(3) with the $H_{n}^{(1)^{\prime}}, H_{n}^{(1)}$, respectively, hence obtaining

$$
\begin{aligned}
& E_{\rho}(\rho, \phi, z=0)=-j \frac{k_{z}}{k_{\rho}} E_{0} H_{n}^{(1)^{\prime}}\left(k_{\rho} \rho\right) e^{-j n \phi}, \\
& E_{\phi}(\rho, \phi, z=0)=-\frac{n k_{z}}{k_{\rho}^{2}} E_{0} \frac{H_{n}^{(1)}\left(k_{\rho} \rho\right)}{\rho} e^{-j n \phi} .
\end{aligned}
$$

Note that Hankel functions are singular along the $z$-axis (i.e., $\rho=0$ ), thus they do not satisfy the homogeneous wave equation. The capability of such inward traveling wave distributions to generate a high-order nondiffracting beam has been demonstrated and discussed in detail in Ref. 33 for a monochromatic Bessel beam; for brevity, the results are not reported here, but numerical and experimental validations of such an assumption for $n=0$ can be found in Refs. 27, 34 .

As for the case of zeroth-order Bessel beams ${ }^{27}$, the unavoidable aperture truncation limits the nondiffracting behavior up to a distance $z_{\mathrm{NDR}}=\rho_{a} \cot \theta(\omega), \rho_{a}$ being the aperture radius and $\theta(\omega)=\arctan \left[k_{z}(\omega) / k_{\rho}(\omega)\right]$ the axicon angle (see Fig. 1). Note that the frequency dependence of the axicon angle results from the nonnegligible wavenumber dispersion which typically affects microwave launchers ${ }^{35}$.We restrict here our attention to the case of $n=1$ and enforce the inward transverse electric field $\mathbf{E}_{t}=E_{\rho} \hat{\mathbf{u}}_{\rho}+E_{\phi} \hat{\mathbf{u}}_{\phi}$ given by Eq. (4) over a finite aperture having a radius $\rho_{\mathrm{a}}=25 \mathrm{~cm} \simeq 10 \lambda_{0}$, with a radial wavenumber $k_{\rho}=0.4 k_{0}$ at the operating frequency $f_{0}=12.5 \mathrm{GHz}$. The electromagnetic field $\mathbf{E}^{\mathrm{rad}}=E_{\rho}^{\mathrm{rad}} \hat{\mathbf{u}}_{\rho}+E_{\phi}^{\mathrm{rad}} \hat{\mathbf{u}}_{\phi}+E_{z}^{\mathrm{rad}} \hat{\mathbf{u}}_{z}$ radiated by this aper- 


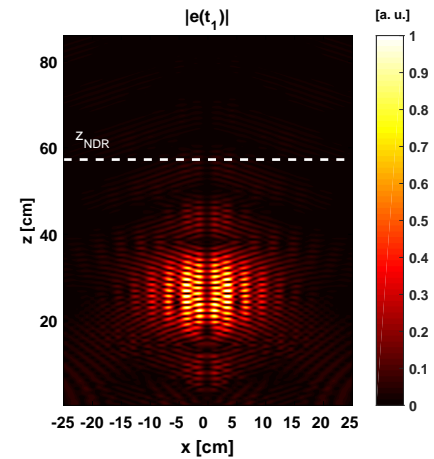

(a)

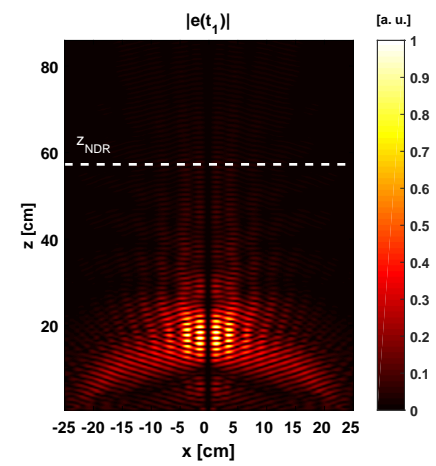

(c)

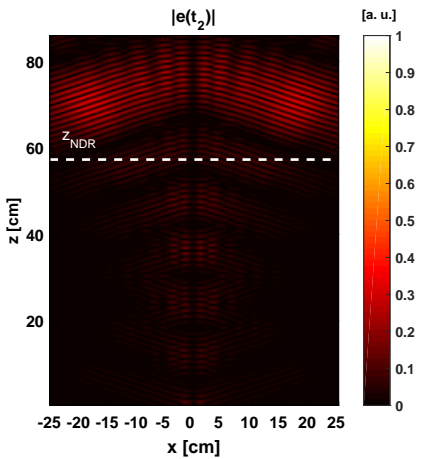

(b)

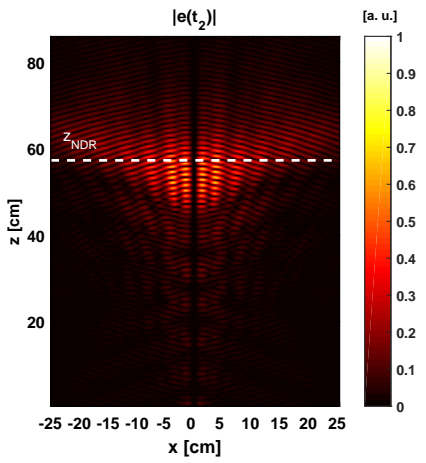

(d)
FIG. 3. XOAM field amplitude in the azimuthal $x z(\phi=0)$ plane. Comparison between (a)-(b) nondispersive and (c)-(d) dispersive case. The intensity of the vectorial electric field $|\mathbf{e}|$ has been reported for two time instants: (a), (c) $t_{1}=0.8 \mathrm{~ns}$ and (b), (d) $t_{2}=2.4 \mathrm{~ns}$. Note that $t_{1}$ and $t_{2}$ correspond to the time instants for which the nondispersive pulse would have traveled a distance equal to $\frac{1}{2} z_{\mathrm{NDR}}$ and $\frac{3}{2} z_{\mathrm{NDR}}$, respectively (Multimedia view). Note that $z_{\mathrm{NDR}}=57.3 \mathrm{~cm}$

ture has been obtained by taking advantage of Huygens' principle $^{6}$. In particular, the equivalent source distribution, i.e., the tangential aperture field at $z=0$ given by Eq. (4), is evaluated and numerically integrated through the standard approach involving the scalar free-space Green's function ${ }^{35,36}$. It is worth noting that such an aperture field can be synthesized by means of microwave radiators such as RLSA antennas ${ }^{27,33}$.

In Fig. 2(a) and (b), amplitude and phase of the resulting monochromatic nondiffracting Bessel beam have been reported for the longitudinal component. As expected a vortex beam is generated, i.e., a higher-order $(n=1)$ Bessel beam.

Once the monochromatic higher-order Bessel beams are generated, a twisted X-wave can be obtained by superposing continuously monochromatic Bessel beams over a certain frequency range. This is accomplished by numerically evaluating the inverse Fourier transform of

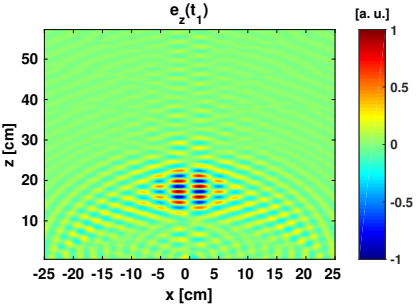

(a)

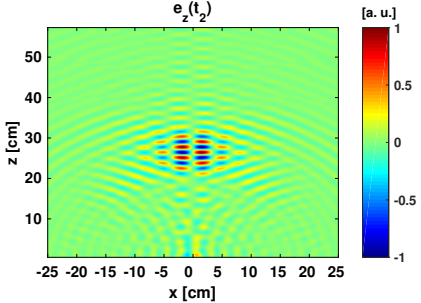

(b)
FIG. 4. XOAM time-domain normalized distribution on the $x z(\phi=0)$ plane of the electric-field component $e_{z}$ inside the nondiffractive range for two time instants: (a) $t_{1}=0.8 \mathrm{~ns}$, (b) $t_{2}=1.2 \mathrm{~ns}$.

the radiated beams $E_{z}^{\mathrm{rad}}$ as follows

$$
e_{z}(\rho, \phi, z ; t)=\int_{-\infty}^{\infty} F(\omega) E_{z}^{\mathrm{rad}}(\rho, \phi, z ; \omega) e^{j \omega t} d \omega
$$

being $F(\omega)$ an arbitrary frequency spectrum.

In a first approximation, the cone dispersion ${ }^{37}$, i.e., the frequency dependence of the axicon angle is usually neglected $\left(\theta(\omega) \simeq \theta_{0}\right)$, as corroborated by experiments in optics or in acoustics ${ }^{24,38}$. As a consequence, the normalized wavenumbers $k_{\rho} / k_{0}=\sin \theta_{0}$ and $k_{z} / k_{0}=\cos \theta_{0}$ are usually assumed to be constant. However, when $E_{z}^{\mathrm{rad}}$ is generated at microwaves over a considerable fractional bandwidth, a nonlinear dispersion of both $k_{\rho} / k_{0}$ and $k_{z} / k_{0}$ must be taken into account for a rigorous derivation of the pulse $\mathrm{p}^{15,37}$. In particular, as already done in Ref. 25, we consider here a second-order Taylor expansion of the radial wavenumber around the operating angular frequency $\omega_{0}$ as follows

$$
k_{\rho}(\omega)=k_{\rho}\left(\omega_{0}\right)+k_{\rho}^{\prime}\left(\omega_{0}\right)\left(\omega-\omega_{0}\right)+\frac{1}{2} k_{\rho}^{\prime \prime}\left(\omega_{0}\right)\left(\omega-\omega_{0}\right)^{2} .
$$

It is worth noting that the wavenumber dispersion is not related to the anomalous dispersion of nonlinear media that has already been exploited for supporting Xwaves $^{37,39-41}$ : here, it is an undesirable phenomenon that has to be taken into account for a realistic description of the spatio-temporal spreading of the field radiated by the aperture. Its impact on the pulse propagation and broadening is discussed in the following.

By considering a uniform frequency spectrum $F(\omega)=1$ with $\omega \in\left[\omega_{0}-\Delta \omega / 2, \omega_{0}+\Delta \omega / 2\right]$ and 0 elsewhere, being $\omega_{0}=2 \pi f_{0}$ and $\Delta \omega$ the bandwidth, the time-domain pulse representation is given by retaining the real part of the positive spectral content of (5), namely $e_{z}(\rho, \phi, z, t)=\Re\left[e_{z}^{+}(\rho, \phi, z, t)\right]$, being $e_{z}^{+}(\cdot)$ the analytic signal. This last expression correctly describes a nondiffracting pulse in the frame of a scalar theory. More generally, the radiated electric field $\mathbf{E}^{\text {rad }}$ leads to the vectorial expression of the localized electric pulse $\mathbf{e}(\rho, \phi, z ; t)=e_{\rho} \hat{\mathbf{u}}_{\rho}+e_{\phi} \hat{\mathbf{u}}_{\phi}+e_{z} \hat{\mathbf{u}}_{z}$ as follows

$$
\mathbf{e}(\rho, \phi, z ; t)=\Re\left[\int_{\Delta \omega} \mathbf{E}^{\mathrm{rad}}(\rho, \phi, z ; \omega) e^{j \omega t} d \omega\right] .
$$




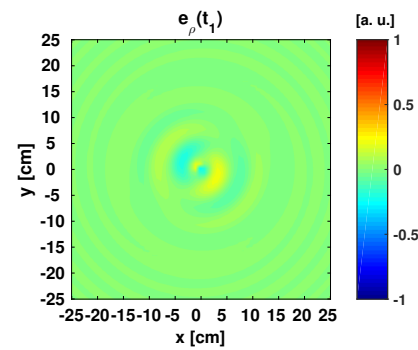

(a)

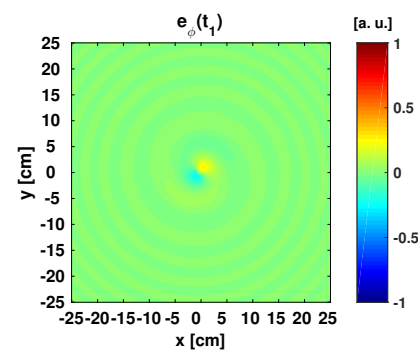

(c)

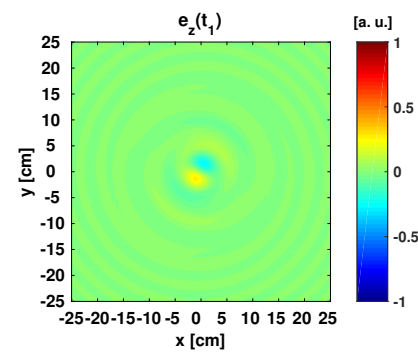

(e)

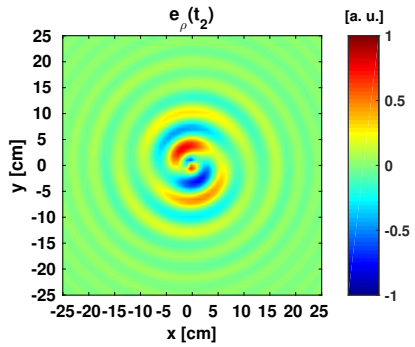

(b)

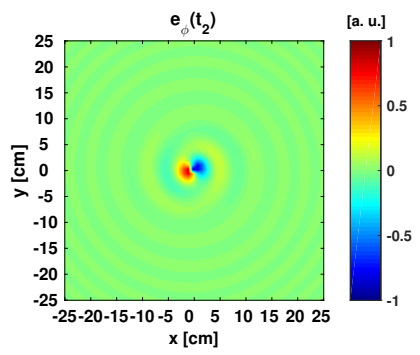

(d)

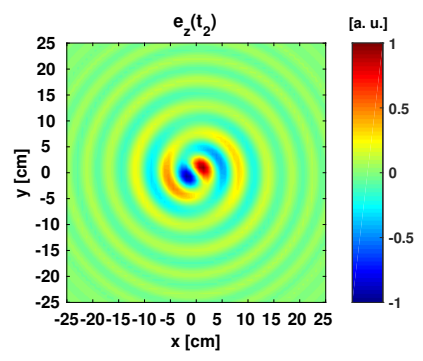

(f)
FIG. 5. XOAM cylindrical electric-field components at $t_{1}=0.8 \mathrm{~ns}(\mathrm{a}),(\mathrm{c}),(\mathrm{e})$, and at $t_{2}=1.2 \mathrm{~ns}(\mathrm{~b}),(\mathrm{d}),(\mathrm{f})$. The transverse plane $z_{0}=z_{\mathrm{NDR}} / 2=28.65 \mathrm{~cm}$ is fixed and time evolution is observed (Multimedia view).

Let us stress that the components of $\mathbf{e}(\rho, \phi, z ; t)$ are still spectral superpositions of Bessel beams, as can be inferred from Eqs. (4). Hence, limited-diffraction pulses are expected to be generated for each component of the electric field. This is corroborated by the numerical results discussed in the following. Note that, for the spectral superposition, a fractional bandwidth $\Delta \omega / \omega_{0}=0.2$ $(\Delta f=250 \mathrm{MHz})$ has been assumed according to the potential wideband capabilities of a RLSA structure ${ }^{42}$. Within the fractional bandwidth, it has been shown ${ }^{25}$ that $k_{\rho}\left(\omega_{0}\right)=\omega_{0} \sqrt{\varepsilon_{r}} / c, k_{\rho}^{\prime}\left(\omega_{0}\right) \simeq\left(0.2-\sqrt{\varepsilon_{r}}\right) / c$ and $k_{\rho}^{\prime \prime}\left(\omega_{0}\right) \simeq 0$, where $\varepsilon_{r}$ is the relative permittivity of the dielectric filling the RLSA and $c$ the light velocity in vacuum.

To assess the effect of the wavenumber dispersion and the diffraction limit, in Fig. 3 the normalized amplitude of the electric field intensity of the pulse $|\mathbf{e}(\rho, \phi, z, t)|$ of the XOAM has been reported at two time instants $t_{1}$ and $t_{2}$ (see the caption for the relevant details) in both the nondispersive (see Figs. 3(a) and (b)) and dispersive case (see Figs. 3(c) and (d)). (The whole time evolution of the dispersive pulse is available online (Multimedia view)). As is clearly visible when dispersion is taken into account, the group velocity is reduced (the distance covered by the pulse is shorter). In addition, the spot size is slightly widened along the transverse direction (see Figs. 3(c) and (d)) without compromising the spatio-temporal localization of the pulse as long as it has not reached the nondiffractive distance $\left(z_{\mathrm{NDR}}=57.3 \mathrm{~cm}\right)$. Conversely, when the pulse is propagating beyond the nondiffractive distance, the twisted pulse is gradually spreading either if the dispersion is (see Fig. 3(d)), or is not taken into account (see Fig. 3(b)). In particular, the spot size progressively grows up and the intensity of the central spot abruptly vanishes. Note that similar results have been obtained for zeroth-order X-waves in Ref. 25. As a consequence, the nondiffractive range also represents the distance for which the OAM is effectively carried by the pulse. In Fig. 4 the longitudinal component of the electric field is reported at the two time instants $t_{1}$ and $t_{2}$ within the nondiffractive range.

Numerical results for the electric field components of the pulse on a transverse plane have been reported in Fig. 5, where their spatial distributions have been evaluated at the reference plane $z=z_{\mathrm{NDR}} / 2=28.65 \mathrm{~cm}$ (see Fig. 1), again for two distinct time instants. Also for this case, the whole time evolution of the dispersive pulse is available online (Multimedia view). The peculiar twisted (i.e., rotating) behavior as well as its nondiffractive nature are visible. The pulse crosses the reference plane around $t_{0}=1 \mathrm{~ns}$ and gradually disappears: it is not yet practically visible at $t_{1}=0.8 \mathrm{~ns}$, whereas it is still crossing the reference plane at $t_{2}=1.2 \mathrm{~ns}$, after that it will be no longer visible. The pulse propagation is quite regular up to the nondiffractive range before the reported time instants (as can be seen in the Multimedia view).

In conclusion, the generation of a monochromatic higher-order Bessel beam carrying OAM has been considered in order to investigate the possibility to generate twisted electromagnetic limited-diffraction pulses at microwaves. The dispersion and radiation properties of RLSA structures have been evaluated for the practical realization of nondiffracting pulses carrying OAM at microwaves. Furthermore, two major issues have been taken into account: i) the generation of higher-order Bessel beams in a wide frequency band and ii) the typical wavenumber dispersion of microwave radiators. The former is obtained by enforcing a traveling-wave rather than a resonant standing-wave illumination, and motivates the use of a $\operatorname{RLSA}^{27,42}$; the latter follows from a realistic description of the RLSA frequency behavior ${ }^{25}$. However, the proposed analysis is general and can be applied to other kinds of microwave radiators characterized by different wavenumber dispersions.

Remarkably, the time-domain analysis has corroborated the attractive features of such configurations, not only for the generation of nondiffracting vortex beams, but also for the generation of nondiffracting twisted 
pulses. As expected, numerical results revealed that, during the propagation, the wavenumber dispersion affects the confinement of the rotating pulse without compromising anyway the limited-diffracting behavior of the original solution up to the nondiffractive distance. This evidence opens interesting scenarios for twisted pulse generation at microwaves, with applications in wireless communications, wireless power transfer, buried-targets detection, and medical imaging.

${ }^{1}$ J. Durnin, J. Opt. Soc. Am. A 4, 651 (1987).

${ }^{2}$ H. E. Hernández-Figueroa, M. Zamboni-Rached, and E. Recami, Localized Waves, Vol. 194 (John Wiley \& Sons, 2007).

${ }^{3}$ J.-Y. Lu and J. F. Greenleaf, IEEE Trans. Ultrason. Ferroelectr. Freq. Control 39, 441 (1992).

${ }^{4}$ P. Schemmel, S. Maccalli, G. Pisano, B. Maffei, and M. W. R. Ng, Opt. Lett. 39, 626 (2014).

${ }^{5}$ M. Ornigotti, C. Conti, and A. Szameit, Phys. Rev. Lett. 115, 100401 (2015).

${ }^{6}$ J. A. Stratton, Electromagnetic Theory (John Wiley \& Sons, 1941).

${ }^{7}$ J. Durnin, J. J. Miceli Jr., and J. H. Eberly, Phys. Rev. Lett. 58, 1499 (1987).

${ }^{8}$ R. Herman and T. Wiggins, J. Opt. Soc. Am. A 8, 932 (1991).

${ }^{9}$ J. Arlt and K. Dholakia, Optics Commun. 177, 297 (2000).

${ }^{10}$ M. Ettorre, S. M. Rudolph, and A. Grbic, IEEE Trans. Antennas Propag. 60, 2645 (2012).

${ }^{11}$ P. Lemaitre-Auger, S. Abielmona, and C. Caloz, IEEE Trans. Antennas Propag. 61, 1838 (2013).

${ }^{12}$ W. Fuscaldo, G. Valerio, A. Galli, R. Sauleau, A. Grbic, and M. Ettorre, IEEE Trans. Antennas Propag. 64, 904 (2016).

${ }^{13}$ J. D. Heebl, M. Ettorre, and A. Grbic, Physical Review Applied 6, 034018 (2016).

${ }^{14}$ E. Recami, Physica A: Statistical Mechanics and its Applications 252, 586 (1998).

${ }^{15}$ H. E. Hernández-Figueroa, M. Zamboni-Rached, and E. Recami, Nondiffracting Waves (John Wiley \& Sons, 2013).

${ }^{16}$ J. N. Brittingham, J. Appl. Phys. 54, 1179 (1983).

${ }^{17}$ E. Heyman, L. Felsen, and B. Steinberg, J. Opt. Soc. Am. A 4, 2081 (1987).
${ }^{18}$ R. W. Ziolkowski, Journal of Mathematical Physics 26, 861 (1985).

${ }^{19}$ R. W. Ziolkowski, I. M. Besieris, and A. M. Shaarawi, J. Opt. Soc. Am. A 10, 75 (1993).

${ }^{20}$ W. A. Rodrigues Jr and J.-Y. Lu, Found. Phys. 27, 435 (1997).

${ }^{21}$ E. Heyman and L. B. Felsen, J. Opt. Soc. Am. A 6, 806 (1989).

${ }^{22}$ A. M. Tagirdzhanov, A. S. Blagovestchenskii, and A. P. Kiselev, J. Phys. A - Math. Theor. 44, 425203 (2011).

${ }^{23}$ J. Salo, J. Fagerholm, A. T. Friberg, and M. M. Salomaa, Phys. Rev. E 62, 4261 (2000).

${ }^{24}$ P. Saari and K. Reivelt, Phys. Rev. Lett. 79, 4135 (1997).

${ }^{25}$ W. Fuscaldo, S. C. Pavone, G. Valerio, A. Galli, M. Albani, and M. Ettorre, J. Appl. Phys. 119, 194903 (2016).

${ }^{26}$ M. Albani, A. Mazzinghi, and A. Freni, IEEE Trans. Antennas Propag. 60, 5538 (2012).

${ }^{27}$ M. Albani, S. C. Pavone, M. Casaletti, and M. Ettorre, Opt. Express 22, 18354 (2014).

${ }^{28}$ A. M. Yao and M. J. Padgett, Advances in Optics and Photonics 3, 161 (2011).

${ }^{29}$ K. Volke-Sepulveda, V. Garcés-Chávez, S. Chávez-Cerda, J. Arlt, and K. Dholakia, J. Opt. B - Quantum S. O. 4, S82 (2002).

${ }^{30}$ I. A. Litvin, A. Dudley, and A. Forbes, Opt. Express 19, 16760 (2011).

${ }^{31}$ D. McGloin and K. Dholakia, Contemp. Phys. 46, 15 (2005).

${ }^{32}$ S. Yan, M. Li, B. Yao, X. Yu, M. Lei, D. Dan, Y. Yang, J. Min, and T. Peng, Phys. Lett. A 379, 983 (2015).

${ }^{33}$ D. Comite, G. Valerio, M. Albani, A. Galli, M. Casaletti, and M. Ettorre, IEEE Trans. Antennas Propag., to appear (2017).

${ }^{34}$ S. C. Pavone, M. Ettorre, M. Casaletti, and M. Albani, Opt. Express 24, 11103 (2016).

${ }^{35}$ C. A. Balanis, Advanced Engineering Electromagnetics (Wiley Online Library, 2012).

${ }^{36}$ R. F. Harrington, Time-Harmonic Electromagnetic Fields (McGraw-Hill, 1961).

${ }^{37}$ M. A. Porras, G. Valiulis, and P. Di Trapani, Phys. Rev. E 68, 016613 (2003).

${ }^{38}$ J.-Y. Lu and J. F. Greenleaf, IEEE Trans. Ultrason. Ferroelectr. Freq. Control 39, 19 (1992).

${ }^{39}$ C. Conti, S. Trillo, P. Di Trapani, G. Valiulis, A. Piskarskas, O. Jedrkiewicz, and J. Trull, Phys. Rev. Lett. 90, 170406 (2003). ${ }^{40}$ C. Conti, Phys. Rev. E 68, 016606 (2003).

${ }^{41}$ A. Couairon, E. Gaižauskas, D. Faccio, A. Dubietis, and P. Di Trapani, Phys. Rev. E 73, 016608 (2006).

${ }^{42}$ M. Ettorre, S. C. Pavone, M. Casaletti, and M. Albani, IEEE Trans. Antennas Propag. 63, 2539 (2015). 